\title{
Rendimiento corporal de especies de peces nativos del Golfo de Fonseca de Honduras
}

Edgar Osiris Carranza Espinal ${ }^{1}$

\section{RESUMEN}

En el Golfo de Fonseca ubicado al sur de Honduras, existen 60 especies de peces de valor comercial. Algunas de estas son clasificadas como pescado blanco (en donde se agrupa el $33 \%$ del total de las capturas). El pescado blanco es de poco valor comercial para los pescadores artesanales, pero con alta viabilidad para el procesamiento primario. El objetivo del estudio fue evaluar los índices del rendimiento corporal de 10 especies de peces nativos del Golfo de Fonseca para identificar las especies con potencial para el procesamiento primario, que consiste en la limpieza y extracción de vísceras para su comercialización y consumo. Se recolectaron ejemplares post captura (con pesos entre 150 y $500 \mathrm{~g}$ ) de peces nativos del Golfo de Fonse$\mathrm{ca}$, ubicados en cuatro comunidades pesqueras. Se registraron medidas morfométricas para calcular los rendimientos de la canal, vísceras, escamas, cabeza, tronco y filete. Se encontraron resultados concluyentes $(\mathrm{P}<0.001)$, siendo el pargo (Lutjanus spp.), la curvina (Cynoscion spp.) y la lisa (Mujil spp.) con el mejor rendimiento de la canal. La especie con el mayor volumen de vísceras fue el jurel $(P<0.001)$. En La palometa (Diapterus peruvianus) se encontró con el mayor peso de la cabeza con relación a su peso total. En el rendimiento del tronco, la lisa, la curvina y el güiche (Ariopsis spp.) presentaron la mayor masa muscular (más del 65\% de su peso corporal). Con respecto al rendimiento del filete, la curvina (Cynoscion spp.), el güiche (Ariopsis spp.) y la pancha rayada (Paralonchurus dumerilii) mostraron más del $38 \%$ de rendimiento, evidenciando que tienen potencial para ser procesados en filete y ser comercializados a un valor más atrayente al mercado.

Palabras clave: pescado blanco, procesamiento primario, rendimiento de filete.

\footnotetext{
${ }^{1}$ Coordinador de la Unidad de Gestión Científica del Departamento de Acuicultura y Biología Marina, Jefe de Laboratorio Húmedo y profesor del Centro Regional Universitario del Litoral Pacífico
} 


\section{ABSTRACT}

In the Gulf of Fonseca located in southern Honduras, there are 60 species of fish of commercial value. Some of these are classified as whitefish (where $33 \%$ of the total catches are grouped). Whitefish is of little commercial value for artisanal fishermen, but with high viability for primary processing. The objective of the study was to evaluate the indices of corporal performance from 10 fish species native to the Gulf of Fonse$\mathrm{ca}$, in order to identify the species with potential for primary processing; which consists in the cleaning and extraction of viscera for commercialization and consumption processes. Post-harvest specimens (with weights between 150 and $500 \mathrm{~g}$ ) were collected from the Gulf of Fonseca's native fish located in four fishing communities. Morphometric measurements were recorded to calculate the yields of the carcass, viscera, scales, head, trunk and fillet. We found conclusive results $(P<0.001)$, being the snapper (Lutjanus spp.), the curvina (Cynoscion spp.), and the mullet (Mujil spp.) with the best carcass performance. The species with the largest volume of viscera was the horse mackerel $(P<0.001)$. In the palometa (Diapterus peruvianus) it was found with the greater weight of the head in relation to its total weight. In the performance of the trunk, the mullet, the curvina and the güiche (Ariopsis spp.) presented the highest muscle mass (more than $65 \%$ of their body weight). Regarding the yield of the fillet, the curvina (Cynoscion spp.), the güiche (Ariopsis spp.), and the striped pancha (Paralonchurus dumerilii) showed more than $38 \%$ yield, evidencing that they have a better potential to be processed into fillet and therefore, are more attractive to the market value

Keywords: white fish, primary processing, fillet yield. 


\section{INTRODUCCIÓN}

La pesca forma parte integral de la economía de muchos países y contribuye a la seguridad alimentaria y nutrición de la sociedad. En el 2014, a nivel mundial, la captura de pescado fue de 93.4 millones de toneladas, registrándose alrededor de 56.6 millones de personas que trabajan en este sector primario (FAO, 2016). En Honduras, la actividad pesquera se desarrolla en las costas del Caribe y el Pacífico, en el Lago de Yojoa y en la Represa Hidroeléctrica Francisco Morazán.

El Golfo de Fonseca situado en la costa pacífica genera alimentos y recursos económicos a través de la pesca artesanal a 43 comunidades costeras (Espinal, 2014). El volumen de captura de peces, crustáceos y bivalvos superan anualmente las 4,090 toneladas métricas (Soto, Andara, \& Sánchez, 2012). Existe una fauna comprendida por 80 especies de peces marinos, 48 de crustáceos, 21 de bivalvos, cuatro gasterópodos y dos de cefalópodos, de las cuales 60 especies de peces son de importancia comercial (DIGEPESCA, 2007).

Los pescadores artesanales categorizan a los peces en pescado blanco y peces de mayor valor comercial. En el caso del pescado blanco, este agrupa el 33\% del total de las capturas anuales y consiste en una mezcla genérica de 11 especies de peces pequeños de carne blanca, cuyo valor de primera venta no supera los U\$D0.40 el Kg (J.Box \& Salgado, 2009). Muchos pescadores consideran este producto de segunda o tercera categoría (y en ocasiones es descartado.)

El pescado blanco es considerado de menor valor comercial por el tamaño del pez, apariencia, estructura ósea, carnosidad, y por ser capturados de forma incidental con el uso de trasmallos al pescar. (Soto, Andara, \& Sánchez, 2012). Las principales especies del pescado blanco son la pancha rayada (Paralonchurus dumerilii), los bagres o güiche (Ariopsis spp.), pancha (Pomadasys spp.), barbudo (Polidactilus spp.), palometa (Diapterus peruvianus), ruco (Haemulon spp.), macarela (Scomberomorus spp.), jurel (Caranx spp.), berrugato (Lobotes pacificus), guabina (Nebris occidentalis) y picuda (Sphyraena ensi).

Las capturas de pescado de mayor valor comercial se cotizan al valor de primera venta entre U\$D 1.70 a U\$D 3.40 el kg, según la estacionalidad del año y la abundancia del recurso (Turcios, 2017), siendo las principales especies la curvina (Scynoscion spp.), pargo (Lutjanus spp.), robalo (Centropomus spp.), lisa (Mugil curema), mero (Epinephelus spp.), tiburón martillo (Sphyrna spp.) y raya (Dasyatis spp.). 
La abundancia del recurso pesquero en el Golfo (Tabla 1) está distribuida en las familias Scianidae con $28 \%$, Carangidae con $13 \%$, Haemulidae con $7.8 \%$ y Ariidae con 7.8\%. Las demás familias se encuentran por debajo del 3\% (Chicas, Gonzáles, \& Ramírez, 2012). Las mayores capturas por la abundancia del recurso son la curvina, la pancha rayada, el jurel, el ruco y los bagres.

Tabla 1. Abundancia del recurso pesquero en el Golfo de Fonseca

\begin{tabular}{|c|c|c|c|}
\hline Familia & Especies & Nombre común & $\begin{array}{c}\text { Abundancia } \\
\text { del recurso \% }\end{array}$ \\
\hline \multirow[t]{2}{*}{ Sciaenidae } & $\begin{array}{l}\text { Cynoscion phoxocephalus } \\
\text { Cynoscion albus } \\
\text { Cynoscion reticulatus } \\
\text { Cynoscion squamipinnis } \\
\text { Cynoscion stolzmanni }\end{array}$ & Curvina/ babosa & $28.0 \%$ \\
\hline & $\begin{array}{l}\text { Paralonchurus dumerilii } \\
\text { Paralonchurus goodei }\end{array}$ & Pancha rayada & \\
\hline Carangidae & $\begin{array}{l}\text { Caranx caballus } \\
\text { Caranx caninus } \\
\text { Gnatanodon speciosus }\end{array}$ & Jurel & $13.0 \%$ \\
\hline Haemulidae & $\begin{array}{l}\text { Haemulon scudderi } \\
\text { Haemulopsis elongatus } \\
\text { Haemulopsis axillaris } \\
\text { Haemulopsis leuciscus } \\
\text { Haemulopsis nitidus } \\
\text { Pomadasys macracanthus } \\
\text { Pomadasys panamensis }\end{array}$ & Ruco & $7.8 \%$ \\
\hline Ariidae & $\begin{array}{l}\text { Ariopsis spp. } \\
\text { Cathorops spp. } \\
\text { Bagre panamensis }\end{array}$ & Güiche/ bagre & $7.8 \%$ \\
\hline \multicolumn{3}{|c|}{ Otras especies comprendida en 15 familias y 59 especies } & $43.4 \%$ \\
\hline
\end{tabular}

Fuente: Chicas, Gonzáles, \& Ramírez, 2012

A pesar de que el volumen de pesca supera las 4,000 toneladas por año, Honduras es uno de los países en donde anualmente se consume de 2 a $5 \mathrm{Kg}$ por persona de pescado, por debajo de la referencia mundial con $18.8 \mathrm{Kg}$ y del consumo aparente de los países industrializados con $26.8 \mathrm{Kg}$. El suministro de la proteína de pescado en la dieta del hondureño es menos de $2 \mathrm{~g}$ por día en comparación a los países industrializados que superan los $6 \mathrm{~g}$ por día (FAO, 2016). En el país, el principal suministro de proteína proviene de la carne. Las carnes rojas y carnes procesadas aportan con 18 g por día, consumiendo $4.5 \mathrm{Kg}$ por persona por año de carne de cerdo y $4 \mathrm{Kg}$ de carne de res (Carranza, 2015). La importancia de motivar el consumo de pescado es 
porque aporta del 15 al $24 \%$ de proteínas de alta calidad y fácil digestión, contiene todos los aminoácidos indispensables (Reyes, 2013), tiene ácidos grasos polinsaturados como eicosapentaenoico y docosahexaenoico, ácidos grasos que previenen enfermedades cardiovasculares y coronarios, diabetes y algunos tipos de cáncer (Gonzáles, Maafs, \& Gómez, 2013). También tiene vitaminas (D, A y B) y minerales como el calcio, yodo, zinc, hierro, potasio, magnesio, cobalto y selenio (Eslava, 2009). El pescado es uno de los alimentos más saludables y tiene una composición nutricional muy completa.

Las especies consideradas pescado blanco como el güiche, pancha, pancha rayada, palometa, ruco, y jurel, son peces que tienen carne clara, buen sabor y textura, son de alto consumo en las comunidades pesqueras, pero la aceptación del mercado nacional es limitada por tener mayor cantidad de espinas y ser de menor tamaño que las otras especies de mayor valor comercial (Martínez, Romero, \& Hernández, 2011). A pesar de las características desfavorables de estas especies, pueden tener potencial en el manejo post-cosecha con el procesamiento primario y fileteado. El objetivo del estudio fue evaluar los índices del rendimiento corporal de 10 especies de peces nativos del Golfo de Fonseca en diferentes intervalos de peso, para identificar las especies con potencial para el procesamiento primario (y ser ofertados en el mercado nacional.)

\section{MATERIALES Y MÉTODOS}

El estudio se realizó en el Laboratorio de Biología del Centro Universitario Regional del Litoral Pacífico (CURLP), en la ciudad de Choluteca, ubicado al sur de Honduras. Se recolectaron 20 ejemplares de 10 especies de peces capturados por pescadores en las comunidades pesqueras de Guapinol, Cedeño, Pueblo Nuevo y San Lorenzo.

Éstos se pesaron en fresco; se almacenaron en hieleras con abundante hielo a la relación 1:1; y se trasladaron al CURLP. Los ejemplares recolectados tuvieron pesos entre los 150 y 500 gramos, debido a la disponibilidad del recurso en los centros de primera venta en las comunidades pesqueras. Las especies que se compararon fueron seis de pescado blanco y cuatro de mayor valor comercial, siendo estos: güiche (Ariopsis spp.), jurel (Caranx spp.), pancha (Haemulopsis spp.), palomenta (Diapterus peruvianus), lisa (Mujil curema), pancha rayada (Paralonchurus dumerilii); además, el ruco (Pomadasys macracanthus), tilapia (Oreocromis spp), pargo rojo (Lutjanus colorado), y curvina (Cynoscion spp.). 
Los peces se lavaron y se pesaron en fresco en una balanza semi-analítica con precisión de $0.1 \mathrm{~g}$ a fin de determinar los rendimientos corporales en función al peso total. A partir de estos pesos fueron determinadas las siguientes variables según la metodología desarrollada por Mora (2005): Peso fresco (PF); peso de escamas (PE); peso fresco sin escamas (PFSE); peso de las vísceras (PV); peso de la canal (PC): [PF (PE+PV)]; peso del tronco (PT): [PC - Peso de la cabeza]; Peso del filete (PFi): [PT Peso del espinazo], A las especies que se les determinó el peso del filete sin piel fueron: la curvina, el pargo, la lisa, la tilapia, jurel y pancha rayada; por ser los peces en los que se observó un mayor peso fresco y una manipulación más fácil en el tratamiento primario .Para la obtención del filete, se realizó un corte manual longitudinal en la musculatura dorsal a lo largo de toda la columna vertebral (con lo que se consiguió dos filetes por pez). El proceso del fileteo fue elaborado por una única persona con experiencia previa.

\section{Rendimiento}

El rendimiento es la cantidad de escamas, vísceras, canal, tronco, cabeza y filete que tienen las diferentes especies de pescado después de ser lavados y procesados, con relación a su peso corporal. La importancia de conocer estos valores es que, para un pescador y un procesador de productos pesqueros, las especies que presentan mayor rendimiento en carne (canal, tronco y filete) son las más atractivas.

Para obtener los respectivos rendimientos se calculó aplicando las siguientes fórmulas:

- Rendimiento de escamas $=($ Peso de las escamas $/$ peso total $) \times 100$

- Rendimiento de vísceras $=($ Peso de las vísceras $/$ peso total $) \times 100$

- Rendimiento en canal $=($ Peso eviscerado $/$ peso total $) \times 100$

- Rendimiento de tronco $=($ Peso del tronco $/$ peso total $) \times 100$

- Rendimiento de cabeza $=($ Peso de cabeza $/$ peso total $) \times 100$

- Rendimiento de filete $=($ Peso de filete $/$ peso total $) \times 100$

\section{Análisis estadístico}

Los indicadores del rendimiento corporal fueron comparados entre clases mediante análisis de la varianza y la prueba de medias Tukey. Los valores porcentuales de rendimiento fueron transformados de acuerdo con la expresión $y=\operatorname{arcsen} \sqrt{ } \times / 100$. Los análisis fueron realizados con el programa estadístico Infostat@2015. 


\section{RESULTADOS Y DISCUSIONES}

Los pesos en el pescado fresco fluctuaron entre 141 y $477 \mathrm{~g}$ entre las especies estudiadas. Los mejores pesos en fresco se observaron en la curvina (Cynoscion spp.), tilapia (Oreocromis spp.), güiche (Ariopsis spp.) y el pargo (Lutjanus colorado). En el peso de la canal se encontraron resultados estadístico significativo $(P<0.001)$, que se observan en la tabla 2. El mejor rendimiento en promedio fue en el pargo (Lutjanus colorado), seguido de la curvina (Cynoscion spp.), la lisa y el ruco (Pomadasys macracanthus). Los peces que mostraron el menor rendimiento en la canal fueron el jurel y la pancha rayada. El rendimiento de la canal consiste en el peso de un animal sin escamas, vísceras y desangrado, previamente de haber sido lavado.

Tabla 2. Peso fresco, peso de canal y rendimiento de la canal de 10 especies nativas de peces del Golfo de Fonseca

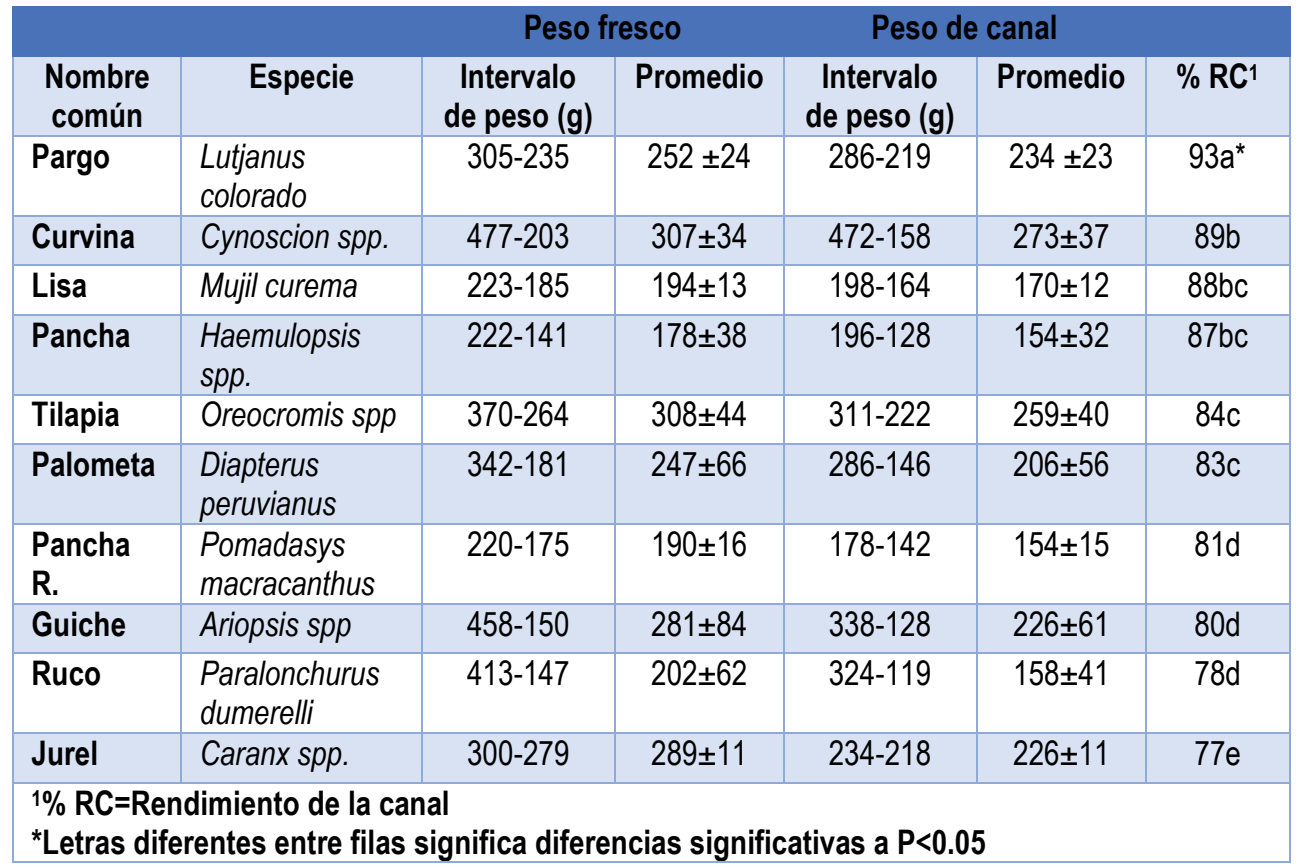

El pargo (Lutjanus colorado) y la curvina (Cynoscion spp.) son especies con alta aceptación en el mercado. La curvina (Cynoscion spp.) es una de las especies de mayor abundancia como recurso pesquero en el Golfo de Fonseca (Soto, Andara, \& Sánchez, 2012). Ambas especies tienen potencial para cultivo. El tamaño del pez influye en el rendimiento de la canal. En el caso de la tilapia, pesa entre los rangos de 
$300-350$ g y 350 a $400 \mathrm{~g}$, el rendimiento de la canal es del 69 y $66 \%$ respectivamente (Rojas, y otros, 2011).

El rendimiento de la canal es muy variable entre las especies, ya que está determinado por la estructura ósea del pez, el volumen visceral y al tamaño de la cabeza. Las especies que tienen alto rendimiento de la canal se debe a la cantidad de la masa muscular en ambos lados de la columna vertebral, que presentan cabeza pequeña y la cavidad abdominal es menor (Kodaira, 2002).

En el rendimiento de las vísceras se obtuvieron resultados concluyentes $(P<0.0001)$. Las especies como el jurel, la tilapia, la pancha y la pancha rayada presentaron entre el 26 y $16 \%$ de su peso corporal en vísceras. El pargo mostró solo el $4 \%$ del peso total (Tabla 3). El volumen de las vísceras depende de los hábitos alimenticios de los peces. Los animales fito-planctívoros cuentan con estómagos pequeños e intestinos muy largos, lo que aumenta la masa vísceral. Por otro lado, Diodatti et al. (2008) comenta que el porcentaje de vísceras se reduce en las fases iniciales del ciclo de vida y aumenta a medida que el pez llega a su madurez sexual, reflejándose en el aumento del peso de las gónadas. Ambos factores influyen en el rendimiento de la canal.

Tabla 3. Rendimiento de vísceras de 10 especies nativas de peces del Golfo de Fonseca

\begin{tabular}{|l|c|c|c|}
\hline \multicolumn{1}{|c|}{ Especie } & $\begin{array}{c}\text { Intervalo de peso } \\
(\mathrm{g})\end{array}$ & Promedio & $\% \mathrm{RV}^{1}$ \\
\hline Caranx spp. & $27-19$ & $23 \pm 3$ & $26 \mathrm{a}^{*}$ \\
\hline Oreocromis spp. & $64-16$ & $29 \pm 15$ & $16 \mathrm{ab}$ \\
\hline Pomadasys macracanthus & $33-23$ & $29 \pm 4$ & $16 \mathrm{ab}$ \\
\hline Paralonchurus dumerelli & $60-9$ & $16 \pm 12$ & $16 \mathrm{ab}$ \\
\hline Ariopsis spp. & $99-22$ & $48 \pm 19$ & $14 \mathrm{~b}$ \\
\hline Cynoscion spp. & $128-17$ & $38 \pm 23$ & $11 \mathrm{c}$ \\
\hline Haemulopsis spp. & $20-10$ & $14 \pm 5$ & $11 \mathrm{c}$ \\
\hline Diapterus peruvianus & $32-14$ & $21 \pm 7$ & $11 \mathrm{c}$ \\
\hline Mujil curema & $18-14$ & $16 \pm 1$ & $8 \mathrm{~cd}$ \\
\hline Lutjanus colorado & $13-9$ & $11 \pm 1$ & $4 \mathrm{~d}$ \\
\hline 1\%RV = rendimiento de visceras & & & \\
\hline *Letras diferentes entre filas significa diferencias significativas a P<0.05 & \\
\hline
\end{tabular}


En el caso del rendimiento de las escamas se encontraron diferencias estadísticas significativas $(P<0.00001)$.Los peces con el menor peso en escamas fueron la pancha (Pomadasys spp.) y la curvina (Cynoscion spp.) con el $1 \%$ de su peso. Los peces que presentaron el mayor volumen de escamas en su cuerpo fueron la lisa (Mujil curema) y la pancha rayada (Pomadasys spp.) con el $4 \%$ de su peso corporal (Tabla 4). El jurel (Caranx spp.) y el güiche (Ariopsis spp.) son peces carentes de escamas y la piel es resbaladiza. El manejo post captura para el procesamiento primario es diferente a los demás peces.

Tabla 4. Rendimiento de escamas de 10 especies nativas de peces del Golfo de Fonseca

\begin{tabular}{|l|l|c|c|c|c|}
\hline $\begin{array}{c}\text { Nombre } \\
\text { común }\end{array}$ & \multicolumn{1}{|c|}{$\begin{array}{c}\text { Especie } \\
\text { fresco }(\mathrm{g})\end{array}$} & $\begin{array}{c}\text { Intervalo } \\
\text { de peso } \\
(\mathbf{g})\end{array}$ & Promedio & $\% \mathrm{E}^{2}$ \\
\hline Jurel & Caranx spp. & $289 \pm 11$ & 0 & 0 & $0 \mathrm{a}^{*}$ \\
\hline Guiche & Ariopsis spp. & $281 \pm 84$ & 0 & 0 & $0 \mathrm{a}$ \\
\hline $\begin{array}{l}\text { Pancha } \\
\text { rayada }\end{array}$ & $\begin{array}{l}\text { Pomadasys } \\
\text { macracanthus }\end{array}$ & $190 \pm 16$ & $2.5-1$ & $2 \pm 1$ & $1 \mathrm{a}$ \\
\hline Curvina & Cynoscion spp. & $307 \pm 34$ & $6-1$ & $3 \pm 2$ & $1 \mathrm{a}$ \\
\hline Pancha & Haemulopsis spp. & $178 \pm 38$ & $5-2$ & $4 \pm 1$ & $2 \mathrm{~b}$ \\
\hline Tilapia & Oreocromis spp. & $308 \pm 44$ & $6-4$ & $5 \pm 1$ & $2 \mathrm{~b}$ \\
\hline Palometa & Diapterus peruvianus & $247 \pm 66$ & $7-4$ & $5 \pm 1$ & $3 \mathrm{c}$ \\
\hline Pargo & Lutjanus colorado & $252 \pm 24$ & $9-5$ & $7 \pm 2$ & $3 \mathrm{c}$ \\
\hline Lisa & Mujil curema & $194 \pm 13$ & $9-6$ & $8 \pm 1$ & $4 \mathrm{~cd}$ \\
\hline Ruco & $\begin{array}{l}\text { Paralonchurus } \\
\text { dumerelli }\end{array}$ & $202 \pm 62$ & $12-4$ & $7 \pm 3$ & $4 \mathrm{~d}$ \\
\hline $\begin{array}{l}\text { 2\%E = rendimiento de escamas } \\
\text { *Letras diferentes entre filas significa diferencias significativas a P<0.05 }\end{array}$ \\
\hline
\end{tabular}

En el rendimiento de cabeza se observaron diferencias significativas $(P<0.003)$. Los pesos promedio de las cabezas fueron entre 130 y $25 \mathrm{~g}$. De las 10 especies, la palometa presentó el mayor tamaño de la cabeza con el $57 \%$ de su peso corporal, seguido del jurel y el pargo con 34 y $31 \%$ respectivamente (Figura 1). Las especies con el menor tamaño de cabeza fueron la curvina (Cynoscion spp.), la lisa (Mujil curema), el güiche (Ariopsis spp.) y la pancha (Haemulopsis spp). El tamaño de la cabeza influye directamente en la masa muscular del pez. Las especies con cabeza grande pueden presentar un bajo rendimiento de la porción comestible con relación al peso total y por ello, existe mayor variabilidad al evaluar los rendimientos corporales.

Con respecto a los pesos promedios, del tronco de los peces se observaron entre 200 y $100 \mathrm{~g}$. Las especies que presentaron el mayor rendimiento del tronco fueron la lisa, 
la curvina (Cynoscion spp.), el güiche (Ariopsis spp) y la pancha (Haemulopsis spp), (Figura 1). Los peces con el menor tamaño del tronco con relación a su peso corporal fueron la palometa (Diapterus peruvianus) y el jurel (Caranx spp.). Mora (2005) encontró un rendimiento del tronco del $65.7 \%$ de la cachama (Piaractus brachypomus) y el $67.8 \%$ de la cachama negra (Colossoma macropomun) que son especies de agua dulce y se aproximan a los rendimientos encontrados en las especies estudiadas.

Figura 1. Rendimiento de cabeza, tronco, vísceras y escamas de 10 especies nativas de peces del Golfo de Fonseca

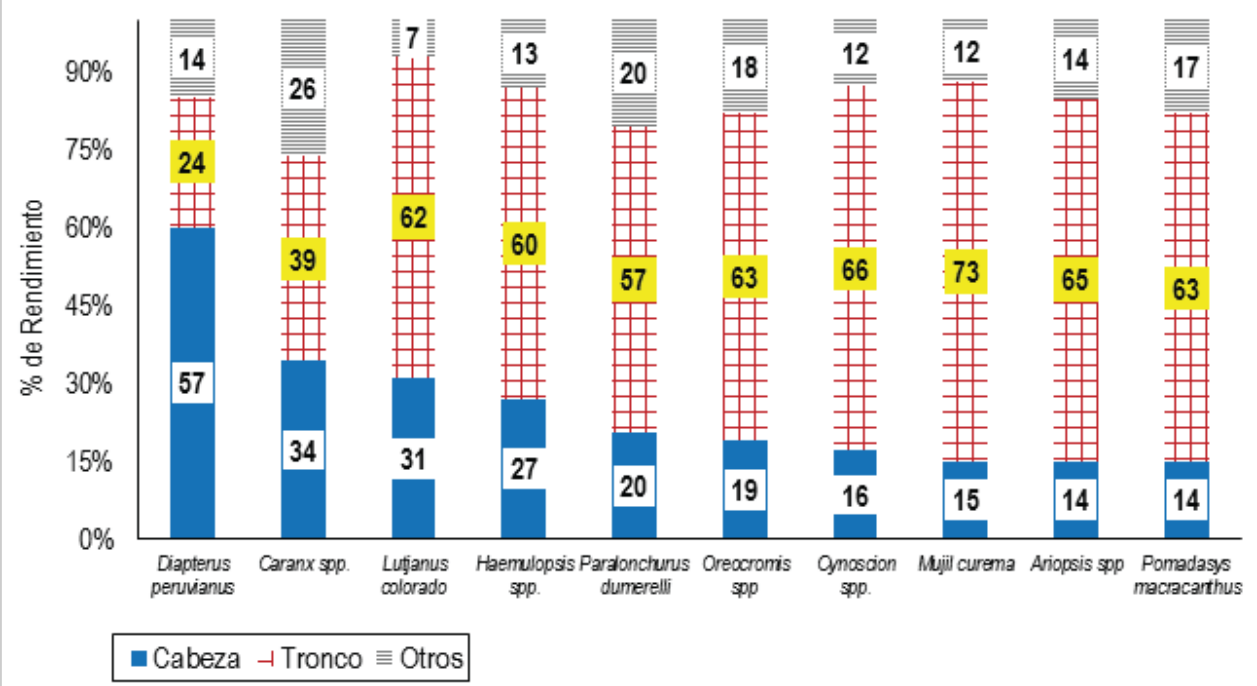

En la tilapia se encontró el rendimiento del tronco en un $63 \%$; este valor coincide los resultados de Rojas et al. (2011) que fueron valores entre 63.7 y $64.1 \%$. Para Oliviera et al. (2015) un valor aceptable de rendimiento del tronco en peces marinos y de agua dulce debe ser entre 60 y $63 \%$. Los peces con mayor rendimiento de tronco pueden presentar un mayor rendimiento de filete (Santaella, Graciá, Peragio, \& Santaella, 2012).

En el rendimiento del filete se encontraron resultados concluyentes $(P<0.0001)$, esto indica que entre especies los beneficios de los rendimientos porcentuales del filete son diferentes. Los pesos de los filetes estuvieron entre $61.1205 \mathrm{~g}$. La curvina presentó en promedio el mejor rendimiento de filete (Tabla 5), seguido del guiche (Ariopsis spp) y la pancha rayada (Pomadasys macracanthus). 
Tabla 5. Pesos y rendimiento de filete de seis especies de peces del Golfo de Fonseca

\begin{tabular}{|c|c|c|c|c|c|}
\hline $\begin{array}{l}\text { Nombre } \\
\text { común }\end{array}$ & Especie & $\begin{array}{l}\text { Intervalo } \\
\text { de peso }(\mathrm{g})\end{array}$ & Promedio & $\% \mathrm{CV}^{1}$ & $\% \mathrm{Rfi}^{2}$ \\
\hline Curvina & Cynoscion spp. & $205.1-87.3$ & $173 \pm 33$ & 19.0 & $43 a^{*}$ \\
\hline Guiche & Ariopsis spp & $178.6-58.5$ & $139 \pm 30$ & 22.0 & $39 \mathrm{~b}$ \\
\hline Ruco & $\begin{array}{l}\text { Paralonchurus } \\
\text { dumerelli }\end{array}$ & $148.7-52.9$ & $103 \pm 34$ & 33.0 & $38 b$ \\
\hline Tilapia & Oreocromis spp & $133.2-95.0$ & $114 \pm 15$ & 13.0 & $36 \mathrm{bc}$ \\
\hline Jurel & Caranx spp. & $93.0-86.5$ & $90 \pm 2$ & 3.0 & $34 \mathrm{~cd}$ \\
\hline Lisa & Mujil curema & $80.3-66.6$ & $70 \pm 5$ & 7.0 & $30 \mathrm{~d}$ \\
\hline Pargo & Lutjanus colorado & $79.3-61.1$ & $65 \pm 6$ & 10.0 & $26 d$ \\
\hline
\end{tabular}

En algunas especies de peces de agua dulce se han encontrado estos rendimientos en el joturo (Joturus pichardi), con $38.35 \%$, cachama negra (Colossoma macropomun) entre el 41.6 y 52\% (Chiquinquirá, Perdomo, Pasquier, García, \& Valecillo, 2011), en el terror verde (Andinoacara rivulatus) entre 27.\% y 33.2\% (Gonzáles, Rodríguez, López, Vergara, \& García, 2016) y en la tilapia este valor es del 38.35\% (Rojas, y otros, 2011). En el caso de las especies de peces nativos del Golfo de Fonseca, como la lisa y el pargo, presentaron los rendimientos más bajos. En las otras especies del estudio, este rendimiento está dentro del rango aceptable.

El rendimiento del filete depende de factores como peso corporal, condición sexual, características morfométricas, técnica de procesamiento, métodos de fileteado y eficiencia del fileteador, siendo este último factor el de mayor incidencia en el rendimiento final del procesamiento para los peces (Rojas, y otros, 2011). Un rendimiento aceptable de los filetes según Eslava (2009) debe ser entre 30 y $35 \%$.

Especies con cabeza más corta y un tronco más largo estima que las dimensiones corporales son más apropiadas para la valoración económica por tener mejor aprovechamiento de su carne durante el procesamiento primario y el fileteado. La comercialización del filete de especies de pescado blanco es una oportunidad que debe impulsarse para obtener mejores beneficios económicos para el pescador, y así se motiva el consumo de la carne de pescado. 


\section{CONCLUSIONES}

Las especies que presentaron el mejor rendimiento de la canal $(P<0.001)$ fueron el pargo, la curvina, la lisa y el ruco. El pargo y la curvina son peces que se evidencia que es muy favorable su comercialización de forma entera, en tallas entre 300 y 450 g. El jurel y la pancha rayada presentaron un rendimiento de la canal menor del $80 \%$. Ambas especies presentaron más del $15 \%$ de su peso total en masa visceral. El rendimiento de la canal en los peces depende de la especie, la estructura ósea, tamaño de la cabeza y volumen de las vísceras.

En la relación cabeza y tronco, la palometa presentó el mayor peso corporal de la cabeza, seguido del jurel y el pargo. Las especies con mejor proporción del tronco fueron la lisa, la curvina, el güiche y la pancha, estas especies presentaron la mayor abundancia de masa muscular, evidenciando que pueden transformarse en productos con valor agregado.

La curvina fue el pez con mejor rendimiento de filete (43\%), seguido del güiche y la pancha rayada con 39 y $38 \%$ respectivamente. Las especies que presentaron los rendimientos más bajo de filete fueron la lisa y el pargo que están por debajo del rendimiento aceptable (30-35\%). Las especies de pescado blanco como el jurel, el güiche y la pancha rayada, así como la curvina, tienen potencial para ser procesados en filete y ser comercializados a un valor más atractivo en el mercado.

Las especies consideradas pescado blanco (son de menor valor comercial) deben valorarse por su importancia en el rendimiento de filete tales como el guiche y el ruco (cuyo rendimiento es mayor que otras especies como el pargo.) Para los pescadores es importante porque puede comercializarlo mejor. Para el consumidor, representa una opción diferente de pescado a un menor precio.

\section{AGRADECIMIENTO}

El autor desea expresar el agradecimiento a estudiantes que cursaron el último año de la carrera de Ingeniería en Ciencias Acuícolas en el año 2017 al colaborar con la recolección, selección y procesamiento primario de las especies estudiadas para desarrollar esta investigación. 


\section{REFERENCIAS BIBLIOGRAFÍCAS}

Carranza, S. (27 de octubre de 2015). Industría cárnica del país no espera efectos negativos por la alerta de la OMS. El Heraldo, pág. 31. Recuperado el 22 de julio de 2017, de http://www.elheraldo.hn/economia/894896-216/industria-c\%C3\%A1rnica-del-pa\% C3\%ADs-no-espera-efectos-negativos-por-alerta-de-la

Chicas, F. A., Gonzáles, J. A., \& Ramírez, W. E. (2012). Ecología básica de los peces del Golfo de Fonseca: bases para el manejo de la pesca artesanal. Escuela de Biología. San Salvador: Universidad de El Salvador.

Chiquinquirá, Y., Perdomo, D. A., Pasquier, G. A., García, D. E., \& Valecillo, O. R. (2011). Evaluación del rendimiento de la canal y del fileteado de la Cachama (Colossoma macropomun). Zootecnia Tropical, 29(3), 361-370.

DIGEPESCA. (2007). Diágnostico pesquero y acuícola. Tegucigalpa: Secretaría de Agricultura y Ganadería. Recuperado el junio de 2017, de file://C:/Users/Edgar\%200siris\%20Carranz/Downloads/DIAGNOSTICO-PESQUERO-Y-ACUICOLA\%20(1).pdf

Diodatti, F., Fonseca, R., Freato, T., Pérez, P., \& Solís, L. (2008). Morphometric Measurements in the Yield of Body Component in Nile of Tilapia (Oreochromis niloticus). Anales de Veterinaria de Murcia, 24(1), 45-55.

Eslava, P. (2009). Estimación del rendimiento y valor nutricional del Besote (Joturus pichardi). MVZ Cordoba, 1(14), 1576-586.

Espinal, A. (07 de abril de 2014). Abundante riqueza marina ofrece el Golfo de Fonseca. El Heraldo, pág. 21.

FAO. (2016). El estado mundial de la pesca y la acuicultura 2016: Contribución a la seguridad alimentaria y nutrición para todos. Roma: Organización de las Naciones Unidas para Alimentación y la Agricultura.

Gonzáles, M. I., Maafs, A. G., \& Gómez, C. G. (2013). Perfil de ácidos grasos de diversas especies consumidos en México. (U. d. Rica, Ed.) Biología tropical, 6(4), 1981-1998.

Gonzáles, M., Rodríguez, J., López, M., Vergara, G., \& García, A. (2016). Estimación del rendimiento de la vieja azul (Andinoacara rivulatus). Revista de Investigación de Talentos, 3(2), 36-42.

J. Box, S., \& Salgado, R. (2009). Evaluación de las prácticas pesqueras en las pesquerías del Golfo de Fonseca: recomendaciones para manejo. Tegucigalpa: SAG.

Kodaira, M. (2002). Experienias en las tecnologías postcosecha de las especies de pescado de Venezuela. VI Congreso Venezolano de Acuicultura, (págs. 42-43). San Cristóbal, Venezuela.

Martínez, J. L., Romero, J. R., \& Hernández, N. Y. (2011). Population parameters of the Pacific flagin mojarra Eucinostomus currani captured by shrimp trawling fishery in the Gulf of California. (U. d. Rica, Ed.) Biología Tropical, 59(2), 889-897.

Mora, J. A. (2005). Rendimiento de la canal en cachama blanca (Piaractus brachypomus) y el híbrido Colossoma macropomun $\mathrm{P}$. brachypomus, procesamiento primario y productos con valor agregado. Bioagro, 3(16), 161-169. 
Oliveira, V. D., Vieira, C., Dessinoni, M., Pimentel, R., Fonseca, R., \& Solis, L. (2015). Using Morphometric Variables in Evaluations of Body of Fish Yields. Journal of Veterinary Medicine and Research, 4(2), 1032-1036.

Reyes, M. J. (2013). Peces de cultivo, composición, comparación con carnes de consumo habitual, ventajas del consumo de pescado. DIAETA, 31(143), 26-30.

Rienzo, J. D., Casanoves, F., Balzarini, M., Gonzalez, L., Tablada, M., \& Robledo, C. (2015). InfoStat versión 2015. Grupo InfoStat. Córdoba, Argentina: Universidad Nacional de Córdoba. Obtenido de www.infostat.com.ar

Rojas, Bárbara, Perdoma, D. A., García, D. E., Gonzáles, M., Corredor, Santos, O. (2011). Rendimiento de canal y fileteado de la tilapia (Oreochromis niloticus) variedad Chiltralada producida en el estado de Trujillo, Venezuela. Zootecnia Trópical, 29(1), 113-126.

Santaella, M., Graciá, C. M., Peragio, M. J., \& Santaella, J. (2012). Evaluación sensorial de diferentes presentaciones comerciales de dorada (Sparus aurata) de acuícultura. Anales de Veterinaria de Murcia, 28, 85-96.

Soto, L., Andara, C., \& Sánchez, E. (2012). Informe de evaluación de la actividad pesquera en el Golfo de Fonseca. AECID. Tegucigalpa: X-media.

Turcios. (29 de Julio de 2017). Los pescadores artesanales en el Golfo de Fonseca. (E. O. Carranza, Entrevistador) 